sie auf diesem ungewöhnlichen Platz verweilten, kratzte sich die eine, auf einem Fusse stehena, mit dem andern am Kopfe.

Auch an anderen für gewöhnlich nicht von ihnen besuchten Orten finden sich manchmal Bekassinen ein. So jagte ich 1896 im Oktober 2 Stück in der Nähe des Strassenteiches von einer ziemlich trocknen, gemähten und mit dem dürren Gras bedeckten Wiese auf, am 28. März 1897, als am Ufer des Ziegelteiches viel Sumpfschnepfen eingefallen waren und einmal 10 Stück fast gleichzeitig abflogen, stiegen von einem mit Strohdünger bedecktem Felde zwei, nach kurzer Zeit vier vom Rande dieses Grundstückes und eine weit drinnen im Felde auf. Auch am 5. April d. J. jagte ich zwei von dem gedüngten Felde fort, und am 19. Sept. desselben Jahres erhoben sich, nachdem vier das versumpfte Teichufer verlassen, gleichzeitig sieben Sumpfschnepfen aus einem an diesen Teich grenzenden Kartoffelfeld. Auch an noch anderen aussergewöhnlichen Stellen fallen sie mitunter ein, so stand am 9. September 1900 eine Bekassine mittags am Grossen Frohburger Teiche ganz frei im seichten Wasser, behielt auch ihren Platz inne, als die dort sich ebenfalls aufhaltenden Krick- und andere Enten unter Rufen aufs Wasser flogen, erst als ich ihr sehr nahe gekommen, bequemte sie sich zum Abfliegen, um ganz in der Nähe wieder einzufallen. Am 28. September 1901 hatte man wegen Streumangel eine versumpfte, meist mit Binsen, Seggen etc. bestandene Uferstelle abgemäht; daselbst hatte sich eine grosse Anzahl Sumpfschnepfen niedergelassen. Nachdem bei meiner Annäherung erst einzelne abgeflogen, tat dies dann gleichzeitig ein Dutzend, dem kurz darauf noch einige folgten.

$\mathrm{Ab}$ und $\mathrm{zu}$ hört man an dem einen oder andern Teich auch eine Bekassine meckern. So tat dies z. B. am 7. Juli 1901 vormittags 1 Exemplar, indem es über dem Strassenteich unter abwechselndem Meckern und Dickerufen umherflog, um endlich unter den letzten Rufen am Teichrande einzufallen.

(Fortsetzung folgt.)

\title{
Entwickelung oder Nicht-Entwickelung?
}

(Replik auf die Ausführungen O. Kleinschmidt's).

\section{Von Wilhelm Schuster.}

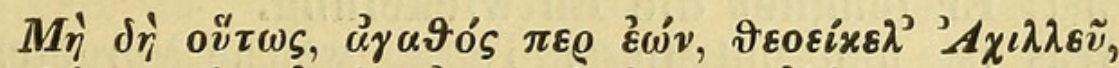

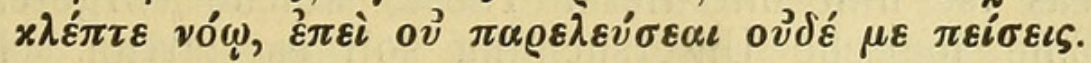

Homer, Ilias I, 131 u. 132.

„O quae mutatio temporum!"

Herr Pfarrer O. Kleins chmidt stellt in No. 12 der „Ornithol. Monatsberichte" 1903 , S. 180 mit besonderem Nachdruck den Satz auf: "Ich protestiere feierlich dagegen, dass die Entwicklungslehre anerkannt sei. Ich erkenne sie nicht an." - Dass der von mir in No. 10 derselben Zeitschrift, S. 156, niedergelegte Passus: 
„Erscheint es nicht wieder auf Grund solcher Erkenntnis als gar sehr fehlerhaft, als kleinlich beschränkt, subtile Artscheidungen in die Natur hineinzutragen nach einem bestimmten „wissenschaftlichen" Schema, einer menschlich subjektiven Schablone - - Artscheidungen, die in Wirklichkeit garnicht vorhanden sind, sondern sich lediglich als Registrierung der Spezifika des (nach Zeit und Ort) anders gearteten Movens von Lebenssitten etc. nur einer Spezies ausweisen? Jener tendenzgetreue Schematismus ist eine Sünde wider die (doch anerkannte) Entwickelungslehre!"6

unmittelbar an die Adresse von Herrn Kleinschmidt ging, welcher vier Seiten vorher (S. 153) auf Grund einer kleinen, an nur fünf sizilischen Habichtsexemplaren (als Parallelstücken zu sizilischen Sperbern) wahrgenommenen sogenannten „Artverschiedenheit" geschrieben hatte: ,Wer da meint, die Natur arbeite nirgends nach einem Schema, der möge diese beiden Formen ansehen und verstummen" - - das war ja eigentlich sehr einfach und klar und wurde auch von Herrn Kleinschmidt ganz richtig herausgefühlt. Aber dass sich Herr Kleinschmidt daraufhin zu der obigen paradoxen These: „Ich erkenne die Entwickelungslehre nicht an" drängen lassen würde, das hätte ich denn doch nicht erwartet. Denn die Leugnung der Entwickelung in der organischen Natur (und damit der Tierveränderungen) ist doch keine conditio sine qua non für die Anfechtung meiner Tatsachenmitteilung (betreffend die veränderte Nistweise der Juister Brandenten); Herr Kleinschmidt hätte doch vielleicht auch noch auf andere Weise mich "totmachen" bezw. mir Widerpart leisten zu können glauben dürfen. Ich erkläre mir also die obige, (wie sich zeigen wird: ganz einzigartige) Idee Kleinschmidts als eine extrem individuelle These, hervorgegangen aus der augenblicklichen oppositionellen Stellung ihres Autors, erzielt im getreuen Verfolg vorausgehender prinzipieller Gedankenaufstellungen: Als das momentane geistige Entwickelungsprodukt aus schon lange vorher (stark) gährenden Tendenzen. Und ob nun Kleinschmidt jetzt auch noch die Entwickelung in der Natur leugnet, um seine Theorien über "Formenkreis" und „Lebensring“" um so eher - sit venia verbo! - „durchdrücken“ zu können oder ob darum nicht, ist mir hier weiterhin Nebensache; Tatsache ist: Er leugnet die Entwickelung.

Was ist nun mit dieser These, welche Vorgeschichte hat sie? Eine logische Untersuchung soll es dartun. Es springt zunächst sofort in die Augen, dass diese These ihrem ganzen Charakter nach auf derselben Linie vorwärtsläuft, auf welcher alle jenen modernen - berufene und unberufene! - Bestrebungen paradieren, welche so etwa das Motto an der Spitze tragen: „Tod dem Darwinismus." Der Geist der Kleinschmidt'schen These ist - jedoch, wie sich später zeigen wird, nur scheinbar identisch mit demjenigen, welcher sich in den gleichfalls neu- 
zeitlichen (in No. 10 des „Zool. Gart." 1903 von mir in einem längeren Essay, S. 325-332, abgefertigten) Totelager-Leichenreden: „Am Sterbelager des Darwinismus" breitmacht. Auf den ersten Blick zunächst anscheinend dieselbe Signatur, derselbe formfertige, geschlossene polemische Typus (und doch nur anscheinend)! Dieser verneinende Geist des Widerspruchs mit seinen so garnicht verfänglichen Produzierungskünsten - ich will nicht sagen: in Mephistopheles' Art - ist zur Zeit auf naturwissenschaftlichem Gebiete ein ungemein aktuelles Thema. Und darum quae mutatio temporum! Vor fünfundzwanzig, ja noch vor fünfzehn Jahren wäre gewisslich die eben hier gekennzeichnete Fechterbezw. Thesenstellung durchaus nicht möglich gewesen. Heute aber ist sie möglich - - weil sich so und so viele akademische Lehrer mit mehr oder minder grossem Vorbehalt, mit geringeren oder stärkeren Modifizierungen, mit engerer oder weiterer, immer Mass und Ziel setzender Reserve [und N. B. auch mit vielleicht mehr oder weniger Recht] gegen den sogenannten „Darwinismus" - den typischen Darwinismus im engeren, beschränkten Sinne (also die Selektions-, Zuchtwahltheorie) - ausgesprochen haben - - nota bene, sich ausgesprochen haben nicht strikte und absolut, sondern unter Anerkennung des Darwin'schen und VorDarwin'schen (Lamark'schen) Gesamtbaues freilich allesamt nur mit engeren oder weiteren Modifizierungen, welche einerseits nur Einzelheiten an dem System ändern wollen und andererseits für jeden einzelnen Thesensetzer eine gewisse, vorsichtigerweise aufgerichtete Deckung bedeuten _- _ _ sich ausgesprochen haben nur gegen den „Darwinismus" im engeren Sinne. Weil ein Wigand, Hamann, Haacke, Driesch, Julius von Sachs, Goette, Korschinsky, Haberlandt, Steinmann, Eimer, Fleischmann (es sind das so ziemlich alle hier event. zu nennenden Namen) gewesen sind, ist die These eines Kleinschmidt möglich. Nur darum! ${ }^{1}$ ) Nun das Aber! Alle diese Männer der Wissenschaft haben sich ja aber niemals gegen die Entwicklungslehre selbst gewandt.

1) Und von diesen wenigen, hier namhaft gemachten Mănnern der Wissenschaft (von den nichtwissenschaftlichen, sondern religiösen Intentionen folgenden Gegnern sehe ich natürlich ab), welche sich irgendwie und irgendwann einmal polemisch gegen Darwin gewandt haben, „möchte sich gewiss wohl ein Teil (so z. B. vielleicht Haacke, Goette etc.) verbitten, dass bestimmte von ihnen geschriebene Sätze aus dem Ganzen herausgerissen und lediglich in dem einen Sinne verwandt werden, der eine Missdeutung leicht zulässt, oder dass sie auf Grund irgendwelcher nebensächlicher Indizien als Gegner des „Darwinismus" aufgeführt werden. Prof. Eimer z. B. ist ebenso für den engeren Darwinismus (Selektions-, Zuchtwahltheorie) als dagegen, d. h. er billigt das eine und weist das andere zurück; er erklärt auch ausdrücklich, dass er sich garnicht unterfange, einem Manne wie Darwin entgegenzutreten" (Zool. Gart.", 1903, S. 337). 
Keiner von ihnen ${ }^{1}$ ) hat die Deszendenztheorie verworfen. Wogegen sie sich aussprechen, das ist eine besondere Modifikation (nämlich die Darwin'sche) zur Entwickelungslehre, das ist der „Darwinismus", eine Erklärung der Art und Weise der Entwickelung, nämlich durch sexuelle Auslese etc. Die Des zendenztheorie (Enwickelungs-, Abstammungslehre) stammt im Wesentlichen von Lamarck, der „Darwin ismus" (Zuchtwahl-, Selektionstheorie) vou Darwin. Hie (bei Darwin) spezielle Zuchtwahltheorie - dort (bei Lamarck etc.) allgemeine Entwickelungs-, Abstammungslehre schlechthin. Der Unterschied zwischen beiden ist ein elementarer. Abgesehen nun von Weismann, Haeckel, Wallace, Huxley, Hehn, Marshall, Pflüger, Lubbock, F. von Wagner, Ranke, Bölsche, Büchner, Boettger, Brehm, Hertwig, Noll, A. und K. Müller, Keller, Spitzer, Ule, Dodel u. s. w. u. s. w. und hundert anderen ebenso bedeutungsvollen, berühmten Namen, welche klar und fest auch für den „Darwinismus" eintreten, so sehen und kennen die oben zuerst genannten wenigen Männer auch alle doch wenigstens die Entwickelung (und damit eben zugleich die Tierveränderungen), erkennen voll und unumwunden die Entwickelungslehre an. Das Gegenteil wäre auch thöricht. Denn absolut kein natürlicher Lebensprozess, kein organisches Werden, Sein und Vergehen in der Welt ist ohne die - neue Werte schaffende und Veränderungen wirkende - Entwickelung zu denken. Die Entwickelungslehre ist allgemein anerkannt, [der , Darwinismus" nicht] Jeder bemerkt, kennt, nennt sozusagen die Entwickelung, der antike Weise und das moderne Schulkind. Livius, der alte Römer, spricht mit überraschender Deutlichkeit von ihr [38, 17: ,,bei Pflanzen und Tieren ist die den Artcharakter aufrecht haltende Vererbung ohnmächtig gegen die durch Boden und Klima (quantum terrae proprietas coelique) bewirkten Veränderungen; alles entwickelt sich vollkommener an dem Orte seines Ursprungs; bei Versetzung auf einen fremden Boden verwandelt es seine Natur nach den Stoffen, die es aufnimmt"]. Herder (in „Über den Ursprung der menschlichen Sprche") und Goethe (in seinem Pflanzenwerk) erkennen sie an. Alexander von Humboldt, der gefeiertste Patriarch unter den Naturforscheru der ersten Hälfte des 19. Jahrhunderts, Karl Ernst von Baer, der Begründer der modernen Embryologie, Johannes Müller, der Begründer der modernen Physiologie, Charles Lyell, der Begründer der modernen Geologie, Thomas Huxley, Alfred Russel Wallace sind ihre entschiedenen Anhänger. Darwin, m. E. der

1) Nur abgesehen vielleicht von dem etwas unbeständigen Fleischmann, welcher zwar in dem ersten Teil seines "Lehrb. der Zoologie" (1896) ganz natürlich auch für die Entwickelungslehre eintritt, in dem 2. Teil nicht mehr ganz. Vergl. über dieses Buch das Urteil Prof. Plate's im „Jahrbuch der Naturkunde“ I, S. 140! 
grösste Gedankenkombinator aller Zeiten, tritt für sie ein. „Entwickelung" ist der oberste Erkenntnisgrund und der erste Lehrsatz der ganzen wissenschaftlichen Welt, fast schon von Einst und ganz sicher von Heute; die Wissenschaft erkennt, erklärt, verteidigt die Entwickelung als ein Grundprinzip alles Werdens in der belebten (und teilweise auch unbelebten) Natur. Und auch die ganze nicht naturwissenschaftliche Wissenschaft (bezw. Welt), auch die orthodox-theologische, gibt heute die Entwickelung $\mathrm{zu}^{1}$ ). Selbst der Verfasser von „Am Sterbelager des Darwinismus“, ein Dr. E. Dennert, Rektor eines evangelischen Knabenpädagogiums, sagt ausdrücklick: Die Entwickelungslehre erkenne ich an (S. 18-25, S. 73, 74) ${ }^{2}$ ); dasselbe betont ganz entschieden z. B. der berühmte Biologe E. Wasmann, Jesuit, welcher selbst mit überzeugender, ja schlagender Akribie nachgewiesen hat, dass die Lomechusen ein Züchtungsprodukt des Freundschaftsinstinkts der Formikagattung (A meisen) sind (vgl. ,Jahrbuch d. Naturkunde"I, S. 139-145,

1) Wie allgemein und unumstritten die Entwickelungslehre auch gerade in der gegenwärtigen Zeit gilt, dafür habe ich in No. 10 des "Zool. Gart." 1903, S. 327 Folgendes angeführt:

,Wer nur einigermassen unser jetziges geistiges Leben kennt, muss von allem eher reden als von einem „Ersterben“ der Darwin'schen Lehren [gemeint sind hier vor allem die Entwickelungslehren]. Das sagen ihm insbesondere drei Umstände:

A.) Es werden fast alle unsere fachwissenschaftlichen Zeitschriften - die Tagesblätter, soweit sie wissenschaftlich erscheinen wollen, von den Fachzeitschriften beeinflusst, natürlich a u ch - mit Darwin'schen Gedanken und Ideen gespeist, mit Untersuchungen in Darwin'schem Geist und Sinne gefüllt. [- "wie sie heutzutage auf den Gassen gepredigt werden," Kleinschm. „0. M.," S. 179-].

B.) Alle Wissenschaften, selbst die Theologie (als Wissenschaft), haben sich den Darwin'schen Entwickelungsgedanken und die Darwin'sche Methode, eine Sache in ihrem Éntstehen, in ihrem natürlichen Werdegang, zu begreifen, angeeignet. Was speziell die Aneignung des Entwickelungsgedankens durch die Theologie angeht, so unterrichte man sich darüber in den derzeit fortlaufenden genialen Naumann'schen „Briefen über die Religion" in der "Hilfe" des bekannten Pfarrers Dr. Naumann, eines geradezu begeisterten Anhängers der Entwickelungslehre, und in dem Buche: „Christentum und Darwinismus (!) in ihrer Versöhnung“ von Pfarrer Dr. phil. Hermann Franke.

C.) Die hervorragendsten Naturforscher der Gegenwart, die tüchtigsten, arbeitsfreudigsten, weitschauendsten Månner unseres Zeitalters sind für den „Darwinismus, "zum wenigsten aber für die Entwickelungslehre alle."

2) Vgl. z. B. „Nun ist es unzweifelhaft, dass in gewissen Fällen sich eine Umwandlung der Formen nachweisen liess, hinsichtlich der Tiere verweise ich z. B. auf die Versuche von Standfuss mit Schmetterlingen, hinsichtlich der Pflanzen auf den Versuch Haberlandts, den ich in diesen Aufsätzen schilderte (s. III.)" (S. 73). 
„Ein Käfer als Zeuge für die Deszendenztheorie“!). Ja selbst sogar die Benediktiner - „Stimmen aus Maria-Laach" (an denen übrigens auch E. Wasmann mitschreibt, vgl. Jahrg. 1903, während sie Herr Pfarrer Kleinschmidt wohl nicht zu lesen bekommt) stehen nicht mehr auf dem Vor-Lamarck'schen Standpunkt (d. i. dem Standpunkt der alt-semitischen Kosmogonie, der bahylonisch-hebräischen Schöpfungsmythen ${ }^{1}$ ). Kurz und gut - die Entwickelungslehre ist ein Grundfaktor der ganzen modernen Wissenschaft. - Ergebn is: Überall ganz uubedingte, aprioristisch selbstverständliche Anerkennung der Entwickelungslehre. Und nur Herr Pfarrer O. Kleinschmidt in Volkmaritz bei Eisleben leugnet die Entwickelung ganz entschieden.

Und so komme ich denn nun auf Grund der bisherigen Erörterungen $\mathrm{zu}$ folgenden zwei Schlüssen:

1.) Herr Pfarrer Kleinschmidt hat Darwinismus und Entwickelungslehre nicht auseinandergehalten, noch nicht auseinanderzuhalten gewusst, sondern beides verwechselt, durcheinandergeworfen; er hat frischfröhlich auf diese übertragen, was nur jenem zukommt. Dies (was aber selbst einem angehenden Zoologen nicht mehr passieren darf, vgl. hierüber in einer späteren Fussnote (unten) das Urteil Prof. Haacke's!) ist mir das Wahrscheinlichste. Oder aber

2.) Herr Pfarrer Kleinschmidt hat seinen Ausspruch bewusst, mit vollem Verständnis und voller Würdigung der niedergeschriebenen Worte, getan. In diesem Falle stellt sich Herr Pfarrer Kleinschmidt mit seiner paradoxen These auf das Gebiet jenseits aller ernstlichen, massgebend wissenschaftlichen Erörterungen.

Ich will das zweite einmal als gegeben setzen. In diesem Falle genügt es mir, lediglich das Ergebnis festzunageln: die strategische Position Herrn Pfarrer Kleinschmidt's liegt ausserhalb der Grenzen aller jetzt gültigen offiziellen Wissenschaft. Was Jahrhunderte erkannt, was die grössten Geister - und N. B. auch das Gros der kleirien und kleinsten - selbstverständlich gefunden, das verwirft Herr Pfarrer Kleinschmidt schlechthin willkürlich mit einem einzigen fettgedruckten Sätzchen. Ich selbst nun halte Herrn Kleinschmidt für bedeutend unter den zeitgenössischen Ornithologen, ja gerade - ich sage in Allem ganz offen meine Meinung - für einen solchen unter uns, welcher sehr kühne (und eben darum mitunter gänzlich unhaltbare) Gedanken hat und

1) Und selbst die Naturanschauung des Altertums dachte, wenn man năher zusieht, entwickelungsgeschichtlich: „Die Erde brachte hervor Lebewesen (toze haarez nephesch chajah) ..."Gen. I, 1,20 (unter der Leitung Gottes). Wer das zu dieser Stelle gehörige Rafael'sche Bild kennt, kann sich an der Sinnenfälligkeit dieses von dem Entwickelungsgedanken recht leicht überzeugen. 
dabei einen wirklich prächtigen Pinsel führt. Beide Gaben, das Geistestalent des Kopfes und das Malertalent der Hand, sind ihm von Gott in reicher Fülle verliehen. Und Selbstständigkeit und Selbstenergie schätze ich am Manne überaus. Ich habe auch in einem zur Zeit im Verlage von Chr. Adolff in Altona erscheinenden, von mir herausgegebenen Vogelwerke den Kleinschmidt'schen Wortbegriff ,Formenkreis" nebenbei empfohlen, freilich nicht in Kleinschmidt's Sinne, sondern in einem ganz anderen, dem meinen. Aber jener Thesensprung Kleinschmidt's - die Verläugnung der Entwickelung - ist denn doch ein grundsätzlich falscher. Vielleicht darf ich hier ein Bild aus dem Jahrmarktsleben (diesem gleicht ja so vielfach unser Arbeitsleben mit seinem Ringen und Streben) zum Vergleiche heranziehen: Je höher und weiter der Seiltänzer auf dem schwanken Seile springt, um so grösser, gefeierter sind seine Leistungen; aber wenn er einmal zu weit springt - - dann eben fällt er. -

Ich komme zum Kleinen und Einzelnen. Über den Beweis per analogiam wollen wir nicht länger streiten. Wenn ich mir diese Bagatelle näher überlege, kann ich in der Tat Herrn Kleinschmidt Recht geben, wie er vielleicht ebensogut bei einigem Nachdenken von seiner Seite aus mir Recht geben könnte. Es ist das eine Sache, bei der es auf die jeweiligen Begleitmomente ankommt. Bei einem consensus totius familiae, ja classis in der bestimmten beregten Frage habe ich den Friderich'schen Beweisschluss per analogiam für eine sola species dieser familia nicht für gewagt gehalten. [In der Philosophie ist ein regelrechter Beweisschluss per analogiam immer rechtskräftig, vollgültig; - wenn die Naturwissenschaft strenger sein will, so ist es mir schon recht!]

An dem von mir mitgeteilten Tatsachenbeispiel und -beweis ändert die gegenteilige Erörterung garnichts. Ich gehe absatzweise vor und bitte die Leser der „O. M.“, S. $177 \mathrm{ff}$ der No. 12 der „O. M.“ 1903 neben diese meine Ausführung zu legen und vergleichsweise die Antwort neben den $\mathrm{zu}$ beantwortenden Absatz zu stellen. Die Widerlegung wird mir überaus leicht, da die gegenteilige Erörterung nicht nur durchweg ziemlich verworrenen Sinnes ist (wenigstens ist sie lange nicht so klar wie meine Darlegung in No. $10 \mathrm{der}$ „O. M.“ 1903) und andererseits keines der im Einzelnen beigebrachten Widerlegungsmo mente der „Protestnote“ Kleinschmidt's (welcher anscheinend, wie ich auf Grund einiger Einwände annehmen zu dürfen glaube, bis jetzt noch nicht sein Hauptstudium auf Entwickelungsgeschichte gerichtet hat $\left.^{1}\right)$ ) einige beweisende $\mathrm{Zugkraft}$ hat.

1) Viel, leider nur allzu viel „neue“ Arten, Vogelnamen etc. hat ja Herr Kl. schon aufgestellt - - diesen Ruhm will, muss ich ihm lassen. In dieser Hinsicht ist er der Doppelgånger von Brehm I. Brehm I zersplitterte die Arten vielfach nur aus Opposition gegen J. F. Naumann; 
Absatz 1 und 2 (S. 177) registrieren Allgemeines oder früher Mitgeteiltes. - Absatz 3. Wenn Herr Kleinschmidt ein Analogon weiss $z u$ der einen Tatsache, dass die ganze Schar der Brandentenpärchen eines grösseren Bezirks durchweg - in allgemein charakteristisch abgeänderter Weise - auf dem blossen Boden nisten anstatt in Löchern und Höhlen etc., so hätte ich gewünscht, dass er dieses Analogon sogleich namhaft gemacht hätte. Ein einzelner oder eventuell auch zweiter, dritter (etwa infoge spezifisch lokaler Wohnungsnot etc.) abgeänderter Fall (wie er sich da und dort in ornithologischen Handbüchern etc. aufgezeichnet findet) beweist natürlich nichts. ${ }^{\mathbf{1}}$ ) Es muss eine ganze mehr oder minder grosse Entenkolonie sein, die ihre Nistweise en bloc abgeändert hat; diese Abänderung muss Jahr für Jahr in derselben allgemein charakteristischen Weise zu bemerken sein und eine rückschlägige Erscheinung (reversio, Darwin „Entstehung der Arten“, 5. Kap.: Gesetze der Veränderungen) darf ebenfalls nicht zu bemerken sein, weder im Ganzen noch eigentlich (als Atavismus) im Einzelnen. Dies ist alles auf Juist der Fall (wo die Abänderung sogar soweit gegangen ist, dass das einzelne Tier nicht einmal mehr von der generell natürlichen Nistweise Gebrauch macht, nämlich die dargebotenen Höhlen verschmäht). Was den Zeittermin der Veränderung betrifft, so habe ich nachgewiesen, dass die Gesamtabänderung auf Juist eine neuzeitliche ist bezw. in die Gegenwart fällt, da die Enten früher solange in Höhlen nisteten, als Kaninchen vorhanden waren. Ein Analogon kenne ich nicht; ich glaube auch nicht, dass es ein solches gibt. Absatz 4 kann ich übergehen. Es gibt tatsächlich auf Juist eine ganze Reihe von Brandentenuestern, welche oben gänzlich offen sind (was ja auch in Absatz 5 selbst zugegeben werden muss). Diese Nester sowie alle anderen, über welchen sich etwa in $3 / 4$ Manneshöhe der Sanddorn wölbt, befinden sich auf dem blossen Erdboden anstatt in Höhlen und Löchern. Alles anormal! Absatz 5. Die Vergleichsparallele mit Sägern und Schellenten

so war es z. B. bei der Baumläufergeschichte, wo Brehm I aus seinen zwei „neuentdeckten" Baumläuferarten auf den kräftigen überzeugenden Einspruch Naumanns hin flugs sehs neue Arten machte (vgl. "Vollst." Vogelfang" und Naumann II, S. 322); teilweise auch geschah es von Brehm I, weil er absolut etwas neues finden zu müssen glaubte; und teilweise geschah es natürlich, weil er überzeugt war, dass es so einzig richtig sei und so unbedingt geschehen müsste. Die Zersplitterungspolitik, welche Herr Kl. hinsichtlich der Arten betreibt, soll natürlich nur ad maiorem gloriam litterarum ornith., nicht etwa suimet ipsius sein.

1) Vergleiche dazu dies: Lindner, Leverkühn u. a. sahen je ein Drosselnest auf dem Erdboden; für den Nistmodus der Singdrossel sind diese vereinzelten Abnormităten ohne Belang; denn nicht alle Drosseln eines ganzen Waldes hatten ja auf die Erde gebaut, sondern nur je eine einzelne. 
ist ein Schlag ins Wasser. Es gibt wohl Vögel, die auf die verschiedenste, aber in dieser Verschiedenheit je immer koustante Weise ihre Nester bauen. Aber bei der Brandente ist dies nicht der Fall. Wo sie nur immer kann, nistet sie in (Erdoder gar Baum-) Höhlen. Sie hält sonst immer nur diesen e in e n bestimmten Nistmodus, welcher einen allseitig und kompakt verschliessenden Charakter der Nistlokalität erfordert, dauernd stricte inne, wenn sie nur kann. Von diesem Nistmodus ist sie generell abgewichen auf Juist. [Überhaupt ist der Vergleich mit Sägern und Schellenten schon a priori nicht geeignet, passend, angemessen, da ja bei diesen Vögeln nur einmal Einzelne ausnahmsweise - , ,auch gelegentlich", Kl., S. 178 nestverändernde Dispositionen treffen, welche event. im nächsten Jahre schon nicht mehr zu bemerken sind.] - Absatz 6 (Behauptung: Die Abänderung ist $\mathrm{zw}$ ang $\mathrm{gs}$ eise herbeigeführt) ist gänzlich ohne Belang. Natürlich, die Nistweise ist durch externe Einflüsse, durch Zwang von aussen „verändert worden“. Aber für die Tatsache der Veränderung ist dies - ob Aktivität oder mehr Passivität vonseiten des Vogels - doch gänzlich einerlei. Ja, wenn wir genau sein wollen, müssen wir sagen: Die Veränderungspotenz liegt latent im Vogel, der Veränderungsanstoss aber und die Veränderungsleitung erfolgt immer nur von aussen. Eben durch (in bestimmten lokalen Grenzen allgemein gleiche und darum dieselben Veränderungshewegungen herausfordernde, erzielende) örtliche und zeitliche Beeinflussungen. Animal agitur! Das Tier ist das veränderungsbewegliche Lebensagens in dem veränderungstreibenden, -leitenden Weltkosmos. Es ist also für das Ganze total einerlei, ob das Individuum der treibende oder getriebene Faktor in dem universal grossartigen Veränderungsprozess ist - - und darum sollte man verständigerweise solche unterwertigen Einwände gegen die Deszendenztheorie wie den obigen garnicht erheben! - Absatz 7. Die Sandverhältnisse sind auf Juist ganz dieselben wie auf Sylt (oder auch Borkum). Auf Sylt etc. bewohnen die Brandenten durchweg die ihnen dort von Tieren oder Menschen gegrabenen bezw. angelegten Höhlen. Auch auf Juist würden sich in den relativ tiefen, sanddorn-bedeckten Muldentälern (oder richtiger: runden Kesseln) der Insel, wo der Wind nicht hinkommt und Höhlen auch mithin garnicht verweht werden können, solche längere Zeit ganz wohl halten. Nicht, weil die Juister Brandgänse etwa sich diese (ja so ungemein tiermässigen!) „sorgenden Gedanken" machen, ob sich auch in einer Sandhöhle gut brüten lasse, brüten sie nicht in Höhlen, sondern ganz einfach darum, weil sie absolut keine Höhlen hatten (vorfanden). Dass Herr Kleinschmidt nicht auf der Insel Juist war, hätte er m. E. nicht zu sagen brauchen; denn wenn er das eigenartige Terrain nur einigermassen gekannt hätte, würde er wohl gewiss seine (wohlgemeinten) Sandspekulationen - Absatz 7, S. 178 - nicht aufgestellt haben. -- 
Absatz 8 lässt meine tatsächliche Beobachtung hypothetisch zu Recht bestehen. - Absatz 9, 1. Teil mutet mich etwas gar naiv an. Mit solchen Einwänden spasste etwa ein Mittelschullehrer zu Anfang der 70 er Jahre gegen die Darwin'schen Ideen. „Nehmen wir an, die Entwickelungslehre währe richtig, so müsste man alle Juister Brandenten fangen und zeichnen, um jede, die etwa von Borkum oder einem anderen Nisthöhlenlande herüberkommt, sofort als gefährlichen Fremdling, der die neue Rasse zu verderben droht, zu erkennen und totzuschiessen." Soviel ich weiss, ist es doch Tatsache, dass alle Vögel so ziemlich genau an dieselben Brutörter zurückkehren bezw. dort verbleiben. Es ist doch ferner eine triviale ornithologische Wahrheit, dass eine geschlossene Vogelkolonie keine Fremdlinge, auch wenn sie von Borkum oder einem ,anderen Nisthöhlenlande" stammen und vielleicht von Herrn Kleinschmidt mit einer modernen Lebensring-Marke gekennzeichnet sind, einlässt. Und es ist doch schliesslich nur allzu - landläufig! - bekannt, dass ein einzelnes irgendwie „,anormales" Tierchen auf die Veränderung einer ganzen mehr oder minder grossen Gesellschaft gar keinen Einfluss ausübt, sondern dass entweder seine Enkelreihe sich dem Ganzen ontogenetisch assimiliert oder aber eventuell das besagte anormale Tierchen selbst (bezw. seine Nachkommenschaft) untergeht. Ich wundere mich einmal darüber, dass diese drei biologischen Tatsachen Herrn Kleinschmidt noch nicht geläufig sind; und zum anderen darüber, dass Herr Kleinschmidt so abfällig von der „Geflügelhoftheorie Darwins" spricht. ${ }^{1}$ ) Haben die Theologen bis jetzt überhaupt einmal eine der grossartigen Geistesschöpfungen Darwins eingehend und gründlich studiert? Ich meine denn doch, dass man, wenn man nur zwanzig Seiten in „Darwin" mit objektiver Aufmerksamkeit gelesen hat, überhaupt nicht noch, wenn man ehrlich sein will, von einer ,Geflügelhoftheorie Darwins" sprechen kann. - Absatz 9, 2. Teil. Dass sich

1) Hier bei dieser merkwürdigen Gedankenzusammenstellung („Geflügelhof-," also Zuchtwahltheorie!) ergibt sich, glaube ich, wieder deutlich, dass Herr Pfarrer Kleinschmidt Darwinismus (Selektionstheorie) und Entwickelungslehre (Deszendenstheorie) durcheinandergeworfen hat. - „Es muss besonders scharf betont werden, dass man den Darwinismus nicht mit der Abstammungslehre verwechseln darf. Der Darwinismus muss allerdings die Abstammungslehre annehmen; wer die Abstammungslehre anerkennt, braucht aber noch lange kein Darwinist zu sein. Es gibt sogar noch Zoologen und Botaniker, die das nicht einmal wissen" (Haacke, „Die Schöpfung des Menschen," S. 465). - Diejenigen Leute (besonders im Lehrer- und Geistlichenstand), welche Darwin so gerne etwas am Zeuge flicken wollen, haben gewőhnlich, wenn man - es ist das eine von mir persőnlich oft gemachte Erfahrung - năher zusieht, noch niemals nur überhaupt oder wenigstens nicht ernstlich in Darwin's epochemachende Werke selbst hineingesehen. 
eine förmlich neue, gute „Art"“ auf Juist bilden werde - niemand unter uns Ornithologen kann so himmelweit dovon entfernt sein, dies zu behaupten oder überhaupt nur zu glauben, wie gerade ich. Ich habe nur auf die lokale Abänderung aufmerksam gemacht (die mir aber, selbst nach modernen Begriffen gemessen, noch lange nicht genügen würde, um eine neue gute "Art" aufzustellen). Ja, ich habe doch gerade auf Grund dessen das Wesen derjenigen Leute getadelt, welche sich bemüssigt sehen, wegen solch feiner, überall zu bemerkender, spezifisch lokaler oder gar individueller Unterscheidungen - wie sie sich auch bei der ,neuen" Habichtsart Astur gentilis arrigonii oder bei dem lang- und kurzbekrallten Baumläufer, bei der sogenannten „Weiden-“ und "Nonnenmeise" (beide = Sumpfmeise), welche sich nach meinen Untersuchungen nur nach Individuen unterscheiden aufzustellen - ich möchte mit einem anderen Autor lieber sagen : - handwerksmässig zurechtzudrechseln. Diese Leute tragen eben subtile, durchaus unvollständige, teilweis willkürlich gesetzte, sogenannte „Artunterscheidungen" in die Natur - oder besser Naturwissenschaft - hinein. Zum Schaden oder wenigstens zur Verwirrung der realen naturwissenschaftlichen Erkenntnisse! - Das sog. Problem der Inzucht macht keine Schwierigkeiten; die Natur wird schon auf irgendeine Weise für die Vollwertigkeit der von ihr gezüchteten Objekte sorgen. - Absatz 10. Meine Beobachtung bleibt eine Gegenwartsbeobachtung ,mit dem Wert der Wirklichkeit," bleibt ,noch die alte wie zuvor sie auch war": Die Juister Brandenten haben ihre $\mathrm{Nistweise} \mathrm{total} \mathrm{und}$ generell abgeändert, nachdem sie durch die Vernichtung der Inselkaninchen - der Feinde der Inselgewächse, welch letztere die Existenz der Insel sichern - bezw. den Wegfall der Nisthöhlen dazu gezwungen worden sind (dies ist die Gegenwartsbeobachtung); veränderte Lebensbedingungen haben nach aller menschlichen Erkenntnis zum unweigerlichen Postulat veränderte Strukturverhältnisse etc.; also müssen und werden auf Juist lokale Abänderungen irgendwelcher Art auftreten (dies ist die apodiktisch rich íge Zukunftsrechnung, nicht -spekulation). ${ }^{1}$ ) - Über die Jahrtausende bezw. Jahrmillionen der Lebensentwickelung wird der verständige Mensch nicht spötteln; er weiss, dass derjenige, welcher seinen Blick nur auf das Gegenwartsleben richtet, also (nach Kobelt, "Verbreitung der Tierwelt") nur auf den erhaltenen Trümmerrest vieler früherer, vorausgegangener Tiergenerationen, wer verachten will, was die Erdgeschichte erzählt, die Geschichte dieser Erde, welche in ihren aufeinandergelagerten Schichten das eigentliche Tierkapital im Verhältnis zum tierischen Gegenwarts-

1) Warum kann Zodda in der Avicula, Giornale ornitologico italiano 1903 (Sienna), für die meisten Vögel Siziliens „Subspezies“ feststellen? Wegen dem entwickelungsmässig abåndernden Inselcharakter dieses rel. abgeschlossenen Gebiets. 
leben als blosse schlichte Zinsen birgt - nimmer und niemals die Wahrheit der Wirklichkeit erkennen kann! - Absatz 11 und 12 habe ich bereits schon besprochen. Ich sehe nicht, was der indische Sperling beweisen soll. Herr Kleinschmidt spricht sogleich darauf von seinem hypothetischen Lebensring, aber mit dem indischen Sperling (G. L., vergl. selbst S. 180) belegt er doch anscheinend wohl garnichts.

Herr Kleinschmidt wirft das Sätzchen hin, dass die Baumnester der Vögel die ursprüngliche Nestform seien. Ich empfehle ihn, behufs besserer Orientierung ,die Nester und Eier der Vögel in ihren natürlichen Beziehungen, ein Beitrag zur Ornithophysiologie und zur Kritik der Darwin'schen Theorien" (besonders Cap. II, „das Nest," S. 36-64) von W. v. Reichenau, meinem hochgeschätzten Freunde (Kustos des Mainzer zoologischen Museums), die „Entwickelungsgeschichte der Natur" von W. Bölsche sowie die grundlegenden umfangreichen, teilweise mehrbändigen $\mathrm{Ab}$ handlungen der Forscher Marsh, Zittel, Dames, Reichenow, Haacke, Vetter, Parker, Fürbringer, Parlow, Mehnert, Haeckel u. s. w. u. s. w. über die Entwickelungs- und Abstammungsgeschichte der Vögel zu studieren - - und dann wieder über die Frage zu debattieren. Die Deszendenz der Nester geht von den selbstbrütenden Nestern (Buschhühner) zu den Bauchbrütern ohne Nest (Pinguine, Alken, Sturmvögel, Fregattvögel etc.), dann den Muldenbrütern (Hühner, Strausse etc.), dann den Loch- oder Höhlenbrütern (Erdpapageien, Königsfischer, Bienenfresser, Spechte etc.) u. s. f. bis zu den Webernestern (Webervögel).

Ich kehre an den Anfang meiner Auseinandersetzung zurück. Ich habe im besten Falle dies zu konstatieren mich gezwungen gesehen: Herr Pfarrer Kleinschmidt nimmt mit der Leugnung der Entwickelung in der Natur eine Position ein, die ausserhalb der Norm alles wissenschaftlichen Erkennens, alles wissenschaftlichen Denkens unserer Zeit fällt; oder aber im anderen Falle: Herr Pfarrer Kleinschmidt hat bis jetzt den Unterschied zwischen Darwinismus und Entwickelungslehre nicht gekannt bezw. berücksichtigt (und hat sich trotzdem doch über die "Unrichtigkeit" der letzteren -- Entwickelungslehre - ein Urteil erlaubt). ${ }^{1}$ )

Mit seinem kurzen fettgedruckten Sätzchen („Ich erkenne die E. nicht an") hat sich Herr Pfarrer Kleinschmidt m. E. bei den allermeisten zünftigen Zoologen so ziemlich selbst ein Urteil gesprochen, das negativ genug ist.

War es die viele Weihnachtsarbeit, die ihn im Reiche der Göttin Hertha oder Nerthus so schlecht sehen liess, so unvorsichtig machte? Ich bin nebenbei auch ein Theologe so gut

1) In diesem Falle müsste ich bedauern, dass von Herrn Kl. so relativ „viel“ im „,neuen Naumann" steht. Doch kann ein schlechter Biologe noch immer ein guter Systematiker sein. 
wie Herr Kleinschmidt und kenne die Übersumme von Pflichten meines werten Herrn Kollega in statu sacerdotii.

NB. Mein Herr Kollege meinte, es gebe doch auch wohl noch andere Beobachter auf Juist und man solle deren Urteil einmal abhören. Nun: Die Herren O. Leege und Sonnemann haben sich schon längst in meinem Sinne ausgesprochen. Das Letzte, was Otto Leege in der diesbezüglichen Frage veröffentlichte, lautet: „Meine vorjährigen Veröffentlichungen über das Offenbrüten der Brandgänse erregten bei manchen Ornithologen Bedenken, und von einer Regel wollten manche nichts wissen. Kein Wunder, denn auf den übrigen Inseln sind $n u r$ vereinzelte Fälle von Freibrüten vorgekommen, da ihnen dort die natürlichen Bedingungen geboten sind. Meine Ausführungen haben inzwischen durch verschiedene Ornithologen, die mich in letzter Brutperiode besuchten, volle Bestätigung [bezw. Bereicherung durch Mitteilung neuer Tatsachen] gefunden (vergleiche: Sonnemann, Ein Pfingstausflug zu Otto Leege nach Juist. Ornith. Monatsschrift 1903, S. 421-429. W. Schuster, Ein eklatantes Beispiel von Veränderung der Artgewohnheit (bezw. lokaler Anpassung). Ornith. Monatsberichte 1903, S. 153-156). . . . . [Interessant ist ferner die ganz wahrscheinlich richtige Vermutung Otto Leege's]: Möglicherweise dauert die Brütezeit in Höhlennestern kürzere Zeit [(21-28 Tage) als bei freien Nestern (35 Tage)], weil die Eier gegen Witterungseinflüsse und Bodenfeuchtigkeit geschützter liegen“ („Ornith. Monatsschrift 1904, S. 110 und 111) - - also wohl ein weiteres Veränderungsmoment, ein neuer Faktor in der Kette der ewigen Entwickelungsreihe!! „Dem Weisen genug“!

Am 1. Januar 1904.

\section{Zugvögel und Florenwechsel.}

Eine faunistische Studie von Fritz Braun-Konstantinopel.

Die Tiergeographie ist keine isolierte Disziplin, sondern hängt mit den übrigen Gebieten der geographischen Wissenschaft aufs engste zusammen. Die Verbreitung aller Geschöpfe, mögen sie von tierischer oder pflanzlicher Nahrung leben, regelt sich nach der Art des Pflanzenwuchses in den betreffenden Ländern, nach dem Vorhandensein bestimmter Nährpflanzen. Diese sind wieder abhängig von dem Klima und der Beschaffenheit der unorganischen Stoffe, die den Boden des Landes bilden. So können wir aus der Tatsache, dass eine bestimmte species hier oder dort vorkommt, wichtige und recht zuverlässige Schlüsse auf den Pflanzenwuchs und das Klima der bezüglichen Örtlichkeiten 


\section{$2 \mathrm{BHL}$ Biodiversity Heritage Library}

1904. "Entwickelung oder Nicht-Entwickelung?" Journal fu

r Ornithologie 52, 431-443. https://doi.org/10.1007/bf02091489.

View This Item Online: https://www.biodiversitylibrary.org/item/101586

DOI: https://doi.org/10.1007/bf02091489

Permalink: https://www.biodiversitylibrary.org/partpdf/142229

\section{Holding Institution}

Smithsonian Libraries

\section{Sponsored by}

Biodiversity Heritage Library

\section{Copyright \& Reuse}

Copyright Status: Public domain. The BHL considers that this work is no longer under copyright protection.

This document was created from content at the Biodiversity Heritage Library, the world's largest open access digital library for biodiversity literature and archives. Visit BHL at https://www.biodiversitylibrary.org. 